\title{
A NEW FAST APPROXIMATE HILBERT TRANSFORM WITH DIFFERENT APPLICATIONS
}

\author{
Abdulnasir Hossen \\ Information Engineering Department, Sultan Qaboos University, P.O.Box 33 Al-Khod, 123 \\ Sultanate of Oman \\ abhossen@squ.edu.om
}

\begin{abstract}
A new and fast approximate Hilbert transform based on subband decomposition is presented. This new algorithm is called the subband (SB)-Hilbert transform. The reduction in complexity is obtained for narrow-band signal applications by considering only the band of most energy. Different properties of the SB-Hilbert transform are discussed with simulation examples. The new algorithm is compared with the full band Hilbert transform in terms of complexity and accuracy. The aliasing errors taking place in the algorithm are found by applying the Hilbert transform to the inverse FFT (time signal) of the aliasing errors of the SB-FFT of the input signal. Different examples are given to find the analytic signal using SB-Hilbert transform with a varying number of subbands. Applications of the new algorithm are given in single-sideband amplitude modulation and in demodulating frequency-modulated signals in communication systems.
\end{abstract}

Key Words: Fast Algorithms, Hilbert Transform, Analytic Signal Processing.

\section{INTRODUCTION}

The subband decomposition idea applied to both FFT $^{[1,2]}$ and $\mathrm{DCT}^{[3,4]}$ is used in this paper for computing the Hilbert transform. The discrete Hilbert transform is related to the discrete Fourier transform, and both of them can be computed using the fast Fourier transform FFT ${ }^{[5]}$. The Hilbert transform of a signal is equivalent to a $\pm 90^{\circ}$ phase shift in all frequency components of the signal. Considering a signal $x(t)$ with Fourier transform $X(f)$, the Hilbert transform of $x(t)$, denoted by $\hat{x}(t)$, is defined ${ }^{[6]}$ :

$$
\hat{x}(t)=\frac{1}{\pi} \int_{-\infty}^{\infty} \frac{x(\tau)}{t-\tau} d \tau
$$

From this equation, we note that the Hilbert transform $\hat{x}(t)$ may be interpreted as the convolution of $\mathrm{x}(t)$ with the time function $h(t)=1 /(\pi t)$. So for a discrete sequence $x(n)$, Eq. (1) can be represented as:

$$
\hat{x}(n)=\sum_{i=0}^{i=\infty} h(i) x(n-i)
$$

Also we know that the convolution of two functions in time domain is transformed into the multiplication of their Fourier transforms in the frequency domain ${ }^{[7]}$. For the time function $1 /(\pi t)$, we have a Fourier transform of -jsgn $(f)$, where $\operatorname{sgn}(f)$ is the signum or sign function defined as

$$
\operatorname{sgn}(f)=\left\{\begin{array}{cc}
1 & f>0, \\
0 & f=0, \\
-1 & f<0 .
\end{array}\right.
$$

Then, the Fourier transform $\hat{X}(f)$ of $\hat{x}(t)$ is given by

$$
\hat{X}(f)=-j \operatorname{sgn}(f) X(f)
$$

The Hilbert transform signal $\hat{x}(t)$ is obtained by taking the inverse Fourier transform of $\hat{X}(f)$. The analytic signal for a discrete sequence $x(n)$ has a one sidedFourier transform since negative frequencies are zero. It can be determined by calculating the FFT of the input sequence, replacing those FFT coefficients that correspond to negative frequencies with zeros, and calculating the inverse FFT of the result. The analytical signal of a real sequence is a complex sequence with a real part, which is the original data, and an imaginary part that contains the Hilbert transform ${ }^{[5]}$.

The following procedure is to be followed to compute the Hilbert transform for a discrete signal ${ }^{[8]}$ :

1. Calculating the FFT $X(k)$ of the input sequence $x(n)$.

2. Creating a vector $h(k)$ whose elements have the values:

$$
h(k)= \begin{cases}1 & k=0, \frac{N}{2} \\ 2 & k=1,2, \ldots, \frac{N}{2}-1 \\ 0 & k=\frac{N}{2}+1, \frac{N}{2}+2, \ldots, N-1\end{cases}
$$

3. Calculating the element-wise product of $X$ and $h$.

4. Calculating the inverse FFT (IFFT) of the sequence obtained in step 3.

5. Taking the imaginary part of the result of the above step.

For narrow-band signals, the subband FFT ${ }^{[1],[2]}$ can be obtained by decomposing the input sequence into two 
bands corresponding to low- and high-pass sequences. The band with the greater energy is transformed while the other band is ignored leading to a fast and approximate FFT. In this work the subband decomposition idea is applied to compute the Hilbert transform.

The organization of the paper is as follows: In the next section, the idea of the subband $\mathrm{FFT}^{[1],[2]}$ is reviewed. Section 3 introduces the basic idea of the fast approximate Hilbert transform with its properties. Section 4 compares the complexity of the subband Hilbert transform with that of the full-band Hilbert transform. The aliasing errors in the subband Hilbert transform are discussed in section 5. Section 6 contains applications of the fast approximate Hilbert transform in analytic-signal generation, in SSBmodulation, and in FM demodulation. In section 7, concluding remarks are given.

\section{SUBBAND-FFT}

In Fig. 1, $\mathrm{a}(n)$ and $\mathrm{b}(n)$ are the low-pass and high-pass filtered versions of $\mathrm{x}(n)$, with $\mathrm{g}(n)$ and $\mathrm{h}(n)$ denoting their factor-2 down-sampled versions, respectively:

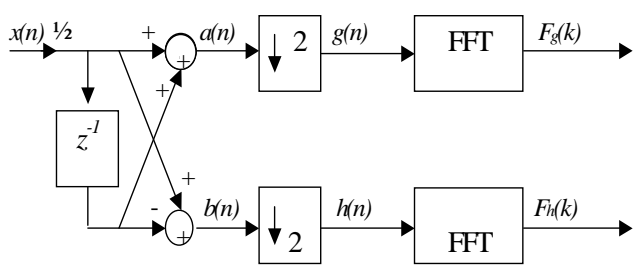

$g(n)=\frac{1}{2}[x(2 n)+x(2 n+1)]$

$h(n)=\frac{1}{2}[x(2 n)-x(2 n+1)]$.

Fig. 1: Two-band decomposition of the subband DFT.

The full-band size- $N$ DFT $X(k)$ can be obtained by

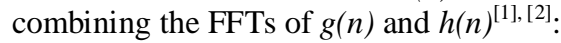

$\mathrm{X}(\mathrm{k})=\left(1+\mathrm{W}_{\mathrm{N}}^{\mathrm{k}}\right) \mathrm{F}_{\mathrm{g}}(\mathrm{k})+\left(1-\mathrm{W}_{\mathrm{N}}^{\mathrm{k}}\right) \mathrm{F}_{\mathrm{h}}(\mathrm{k})$.

Equation (5) is approximated for calculating only the low-pass band:

$$
X(k) »\left(1+W_{N}^{k}\right) F_{g}(k), \quad k \hat{\mathrm{I}}(0,1, \mathrm{~K}, N / 4-1) .(6)
$$

The decomposition process in Fig. 1 can be repeated $\mathrm{m}$ times to get $M=2 m$ subbands, out of which only one band is to be computed depending on information known or derived adaptively ${ }^{[9]}$ about the input signal power distribution. Two types of approximation errors (linear distortion and aliasing) are resulted from the decomposition and approximation processes ${ }^{[2]}$.
Substituting $F g(k)$ in Eq. (6) by the DFT of $g(n)$, and taking $g(n)$ from Eq.(4), we get:

$$
\hat{X}(k)=\frac{1}{2}\left(1+W_{N}^{k}\right) \stackrel{\circ}{\mathrm{a}}_{n=0}^{N / 2-1}(x(2 n)+x(2 n+1)) W_{N / 2}^{k n} .
$$

Equation (7) can be also written as:

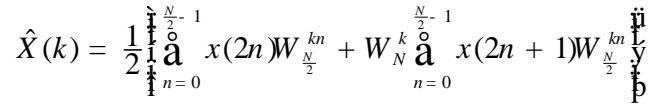

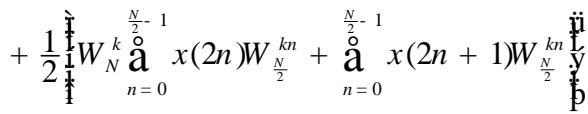

The two types of approximation errors in $\hat{X}(k)$ can be taken into account in the following equation ${ }^{[2]}$ :

$$
\hat{X}(k)=A_{0} X(k)+A_{1} X(k+N / 2)
$$

where $A_{0}$ and $A_{l}$ are two coefficients relating to linear distortions in the exact transform $X(k)$ and aliasing error due to $X(k+N / 2)$, respectively. Noticing the first half of Eq. (8) shows that it is the exact transform $X(k)$ multiplied by a factor of $1 / 2$. The value of $X(k+N / 2)$ can be found from $X(k)$ by replacing each $k$ in $X(k)$ with $k+N / 2$. Now substituting for $X(k)$ and $X(k+N / 2)$ into Eq. (9) yields:

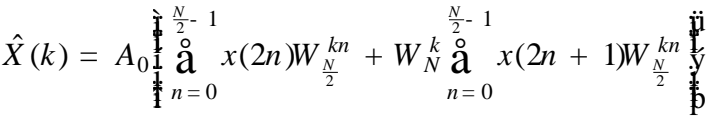

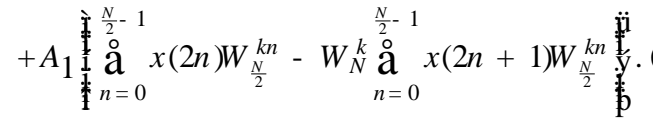

By comparing Eqs. 8 and 10 we obtain the following:

$$
\begin{aligned}
& A_{0}+A_{1}=\frac{1}{2}\left(1+W_{N}^{k}\right), \\
& A_{0}-A_{1}=\frac{1}{2}\left(1+W_{N}^{-k}\right) .
\end{aligned}
$$

Solving Eqs. 11 and 12 yields:

$A_{0}=\frac{1}{2}(1+\cos (2 k p / N))$,

and

$A_{1}=-\frac{j}{2} \sin \left(\frac{2 k p}{N}\right)$

Thus the one-stage approximation in Eq. (6) can be expressed as

$$
\hat{X}(k)=\frac{1}{2}\left[1+\cos \left(\frac{2 k p}{N}\right)\right] X(k)-\frac{j}{2} \sin \left(\frac{2 k p}{N}\right) \mathrm{X}\left(k+\frac{N}{2}\right)
$$

where $\hat{X}(k)$ is the approximate, and $X(k)$ is the true signal spectrum.

For $M=2^{m}$ bands of decomposition, there will be $M-1$ aliasing terms, uniformly distributed on the frequency axis. For the case of retaining only the low-low band 
out of $M=4$ subbands in the approximation, Eq. (9) can be written ${ }^{[2]}$ :

$$
\begin{aligned}
\hat{X}(k) & =A_{0} X(k)+A_{1} X\left(k+\frac{N}{4}\right) \\
& +A_{2} X\left(k+\frac{N}{2}\right)+A_{3} X\left(k+\frac{3 N}{4}\right),
\end{aligned}
$$

And the four aliasing coefficients are found to be:

$$
\begin{aligned}
& A_{0}(k)=\frac{1}{4}\left[1+\cos \left(\frac{2 k p}{N}\right)\right]\left[1+\cos \left(\frac{4 k p}{N}\right)\right], \\
& A_{1}(k)=\frac{1-j}{8} \sin \left(\frac{4 k p}{N}\right)\left[1+\cos \left(\frac{2 k p}{N}\right)-\sin \left(\frac{2 k p}{N}\right)\right], \\
& A_{2}(k)=\frac{-j}{4} \sin \left(\frac{2 k p}{N}\right)\left[1+\cos \left(\frac{4 k p}{N}\right)\right], \\
& A_{3}(k)=\frac{-(1+j)}{8} \sin \left(\frac{4 k p}{N}\right)\left[1+\cos \left(\frac{2 k p}{N}\right)+\sin \left(\frac{2 k p}{N}\right)\right] .
\end{aligned}
$$

\section{SUBBAND HILBERT TRANSFORM}

The subband Hilbert transform is obtained using the following steps:

1. Calculating the SB-FFT $X$ of the input sequence, this results in a sequence of length $L=\frac{N}{2}$ for half-band case, $L=\frac{N}{4}$ for quarter-band case, or generally $L=\frac{N}{M}$ for $M$ sub-bands.

2. Creating a vector $\mathrm{h}$ with length $L$ having the values:

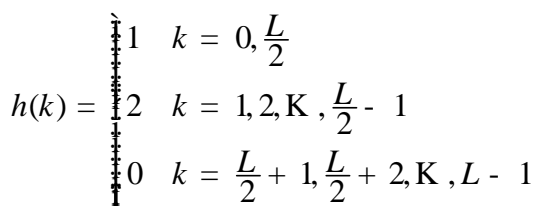

3. Calculating the element-wise product of $X$ and $h$

4. Calculating the IFFT of length $L$ for the sequence obtained in step 3 .

5. Taking the imaginary part of the result of step 4.

All properties known for the full-band Hilbert transform are applicable also to the subband Hilbert transform if the signal to be transformed is a narrowband signal. Some of these properties are investigated in this section with simulation examples. In all these examples the input signal is plotted with solid-line and the output signal is plotted with dotted-line.

1. The Hilbert transform of a constant is zero. Fig. 2 shows that both full-band and half-band Hilbert transforms of a constant is zero.

2. The Hilbert transform of the cosine function $\cos \left(2 \pi f_{c} n T\right)$ is equal to $\sin \left(2 \pi f_{c} n T\right)$. Where $T$ is the sampling interval. Similarly, the function $\sin \left(2 \pi f_{c} n T\right)$ has a Hilbert transform equal to $\cos \left(2 \pi f_{c} n T\right)$. Both the full-band and the SB-Hilbert transformation produce this results, as shown in Fig. 3
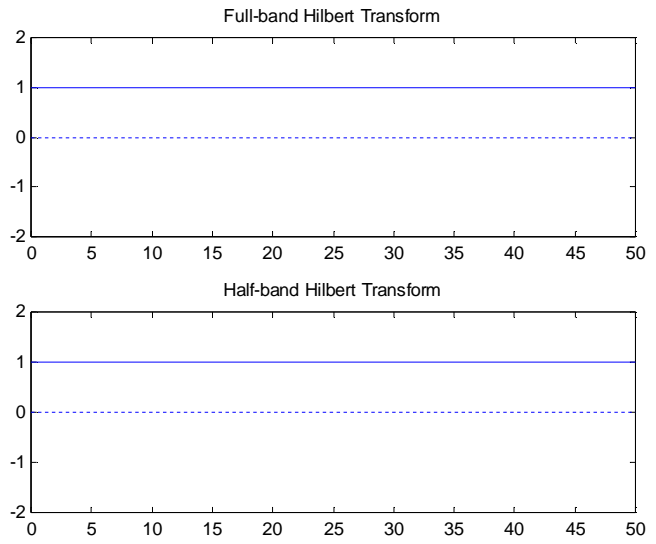

Fig. 2: Hilbert of a constant.

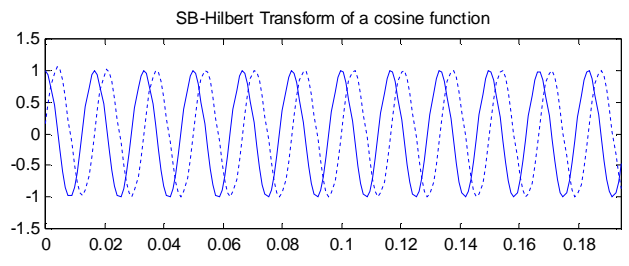
SB-Hilbert Transform of a sine function

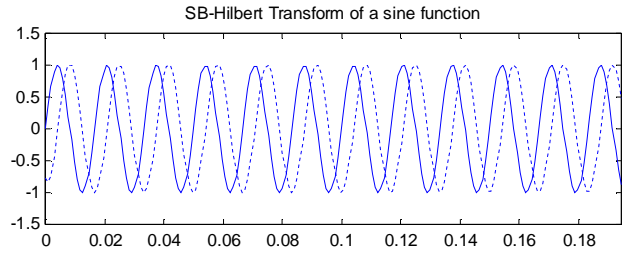

Fig. 3: Hilbert of a cosine function.

3. The energy content of a zero-mean signal is equal to the energy content of the Hilbert transform. The property is tested by finding the energy of a sinusoidal signal (used in property 2) and the energy of its full-band Hilbert transform and subband Hilbert transform with $M=2$ and $M=4$ and $M=8$. It is found that the energy is almost the same in all cases depending on the approximation quality of the subband realization.

4. The signal $x(t)$ and its Hilbert transform are orthogonal. For the same example used to prove the previous two properties, it is proved that, if $\hat{x}(t)$ is used as a full-band Hilbert transform, then:

$$
\underset{-¥}{¥} x(t) \hat{x}(t) d t=0
$$

and the result is almost zero if subband Hilbert transforms of $M=2$ or $M=4$ or $M=8$ are used.

5. The Hilbert transformation of a Hilbert transform produces a phase shift of $\pi$ to the original signal. In Fig. 4, a sinusoidal signal of $50 \mathrm{~Hz}$ is applied to two stages of Hilbert transform. In each stage either a full-band or a half-band Hilbert transform is used. 

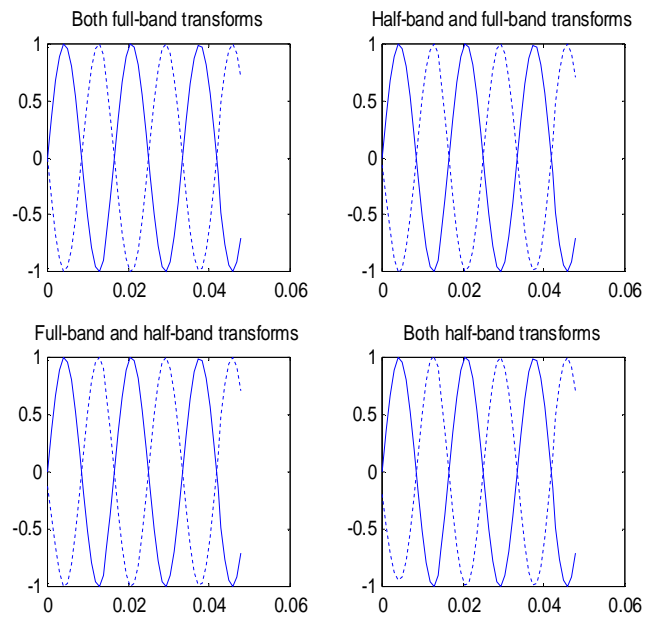

Fig. 4: Hilbert of Hilbert transform.

6. The Hilbert transform is a linear process. An input data sequence $x(n)$ of length 1024 points is used with three impulses as follows:

$$
x(n)=d(n-25)+1.5 d(n-50)+2 d(n-75)
$$

Knowing that the Hilbert transform of $\delta(\mathrm{t})$ is $a / p t$. Both full-band and SB-Hilbert transforms results in Fig. 5 prove the linearity theorem.

\section{COMPLEXITY}

The advantage of using subband decomposition transforms is the reduction of the complexity in comparison to full-band ${ }^{[1-4]}$. Reductions in complexity are obtained from all the steps of section 3. by using sequences of length $\mathrm{L}$ instead of $\mathrm{N}$. The subband Hilbert transform is compared in computational complexity with the full-band Hilbert transform. Table 1 shows the percentage reduction in complexity (measured as execution time) of the Hilbert transform, if SB-Hilbert transforms with different $\mathrm{M}$ are to be used instead of full-band transform.
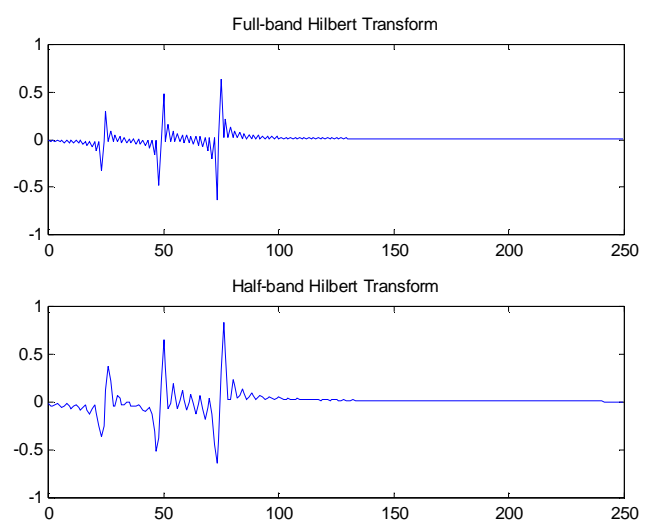

Fig. 5: Linearity Property of Hilbert Transform.
Table 1: Complexity reduction results.

\begin{tabular}{|c|c|c|c|c|c|}
\hline \multirow{2}{*}{ M } & \multicolumn{5}{|c|}{ Reduction in Complexity } \\
\cline { 2 - 6 } & \multicolumn{5}{|c|}{ Number of Input Points } \\
\cline { 2 - 6 } & 64 & 128 & 256 & 512 & 1024 \\
\hline 2 & $23.01 \%$ & $25.91 \%$ & $26.43 \%$ & $28.93 \%$ & $31.48 \%$ \\
\hline 4 & $48.11 \%$ & $50.45 \%$ & $52.81 \%$ & $54.82 \%$ & $56.55 \%$ \\
\hline 8 & $63.58 \%$ & $65.61 \%$ & $67.42 \%$ & $69.14 \%$ & $70.73 \%$ \\
\hline 16 & $75.09 \%$ & $76.38 \%$ & $77.68 \%$ & $79.01 \%$ & $80.22 \%$ \\
\hline
\end{tabular}

\section{ACCURACY}

For $N=16$ and $M=2$ and if only the first band is calculated, Table 2 shows the values of the computed fast approximate (with aliasing errors) half-band Hilbert transform $\hat{x}(n)$, assuming that the ratio of the frequency-transform points causing aliasing to the true components in the calculated band is fixed to $\alpha$.

The values in Table 2 can be computed by calculating the aliasing errors of the SB-FFT and then finding the Hilbert transform of their time signal. The following numerical example describes this interesting result for $N=8$ and $M=2$ :

1. For a case of no aliasing error, a frequency signal of $(1,1,0,0,0,0,0,1)$ is assumed. The half-band Hilbert transform of the first two-points (the lowband case) is found to be 0 and 0.5 respectively.

Table 2: Approximate half-band Hilbert transform with aliasing errors.

\begin{tabular}{|c|c|c|c|c|}
\hline$\alpha$ & $\hat{\mathrm{x}}(0)$ & $\hat{\mathrm{x}}(1)$ & $\hat{\mathrm{x}}(2)$ & $\hat{\mathrm{x}}(3)$ \\
\hline 0 & 0 & 0.5 & 0 & -0.5 \\
\hline 0.1 & -0.0457 & 0.525 & -0.0043 & -0.475 \\
\hline 0.2 & -0.0914 & 0.55 & -0.0086 & -0.45 \\
\hline 0.3 & -0.1371 & 0.575 & -0.0129 & -0.425 \\
\hline
\end{tabular}

2. If an aliasing component $\alpha$ of 0.1 is assumed outside the low-frequency band, the corresponding frequency signal will be: $(1,1,0.1,0.1,0.1,0.1,0.1,1)$. The half-band Hilbert transform of the first two-points is found to be 0.0457 and 0.525 respectively.

3. Comparing the results of $1 \& 2$, the aliasing errors in the Hilbert transform of these two points are 0.0457 and 0.025 respectively.

4. The result in the last step can be found by finding first the aliasing errors in the subband-FFT from Eq. (15). These errors are found to be $(0,-0.0414 j$, $-0.1 j,-0.2414 j)$ for $(k=0,1,2,3)$ respectively.

5. The Hilbert transform of the corresponding time sequence of this error signal is found to be: 0.0457 and 0.025 for the first two points. The values of the aliasing errors found from steps 3 and 5 are the same.

The following numerical example describes the analysis of the aliasing errors in quarter-band SBHilbert transform for $N=16$ and $M=4$ : 
1. For a case of no aliasing error, a frequency signal of $(1,1,0,0,0,0,0,0,0,0,0,0,0,0,0,1)$ is assumed. The quarter-band Hilbert transform of the first twopoints (the low-low-band case) is found to be 0 and 0.125 respectively.

2. If an aliasing component of 0.1 is assumed outside the low-low-frequency band, and the frequency signal is represented as $(1,1,0.1,0.1$, $0.1,0.1,0.1,0.1,0.1,0.1,0.1,0.1,0.1,0.1,0.1,1)$, the quarter-band Hilbert transform of the first twopoints is found to be -0.0165 and 0.1328 respectively.

3. Comparing the results of $1 \& 2$, the aliasing errors in the Hilbert transform of these two points are 0.0165 and 0.0078 respectively.

4. The result in the last step can be found by finding first the aliasing errors in the-FFT from Eq. (17). These errors are found to be:

$(0,-0.0153 j,-0.354 j,-0.077 j)$ for $(k=0,1,2,3)$ respectively.

5. The Hilbert transform of the corresponding time sequence of this error signal is found to be: 0.0165 and 0.0078 for the first two points, and this result is the same as that found in step 3 of this example.

Interpolating the resulting length- $N / 2$ half-band SBHilbert transform by a factor of 2 , leads to a length- $N$ approximated Hilbert transform $\hat{x}(n)$. Table 3 shows the values of the full-band Hilbert transform with $N=16$ and half-band $(M=2)$ subband Hilbert transform with and without interpolating the results by a factor of 2. The input signal has a frequency transform of: $(1,1,1,1,0.1,0.1,0.1,0.1,0.1,0.1,0.1,0.1,0.1,1,1,1)$.

Results show how well the subband Hilbert transform values approximate those of the full-band Hilbert transform.

Table 3: Interpolating the approximated Hilbert transform results.

\begin{tabular}{|c||c|c|c|}
\hline Index: $n$ & Full-band & Half-band & Half-band Inter. \\
\hline 0 & 0 & -0.0223 & -0.0223 \\
\hline 1 & 0.2894 & & 0.2289 \\
\hline 2 & 0.2716 & 0.3122 & 0.3122 \\
\hline 3 & 0.1591 & & 0.1717 \\
\hline 4 & 0 & -0.0011 & -0.0011 \\
\hline 5 & -0.0103 & & -0.0127 \\
\hline 6 & 0.0466 & 0.0539 & 0.0539 \\
\hline 7 & 0.0699 & & 0.0541 \\
\hline 8 & 0 & -0.0006 & -0.0006 \\
\hline 9 & -0.0699 & & -0.0518 \\
\hline 10 & -0.0466 & -0.0497 & -0.0497 \\
\hline 11 & 0.0103 & & 0.0109 \\
\hline 12 & 0 & -0.0011 & -0.0011 \\
\hline 13 & -0.1591 & & -0.1602 \\
\hline 14 & -0.2716 & -0.2914 & -0.2914 \\
\hline 15 & -0.2894 & & -0.2289 \\
\hline
\end{tabular}

\section{APPLICATIONS}

\subsection{Analytic Signal}

There are many applications in communication where it is necessary to perform a $90^{\circ}$ phase shift to the frequency components comprising a signal. One common application is the generation of the complex-valued analytic signal $\ell(n)$. Letting $\hat{x}(n)$ be the Hilbert transform of $x(n)$ with all frequency components shifted by $90^{\circ}$, the analytic signal is given by:

$\tilde{x}(n)=x(n)+j \hat{x}(n)$

So if $x(n)=\cos \left(\omega_{0} n T\right), \quad \hat{x}(n)$ will be $\sin \left(\omega_{0} n T\right)$ and $\tilde{x}(n)$ will be $\cos \left(\omega_{0} n T\right)+j \sin \left(\omega_{0} n T\right)$, which corresponds to only the positive-frequency component of $x(n)$. In Fig. 6, both $x(n)$ (solid line) and $\hat{x}(n)$ (dotted line) are shown for full-band Hilbert transform and subband Hilbert transform with three different values of $M$. In all cases the fundamental frequency is $30 \mathrm{~Hz}$, and the sampling frequency is $1024 \mathrm{~Hz}$.
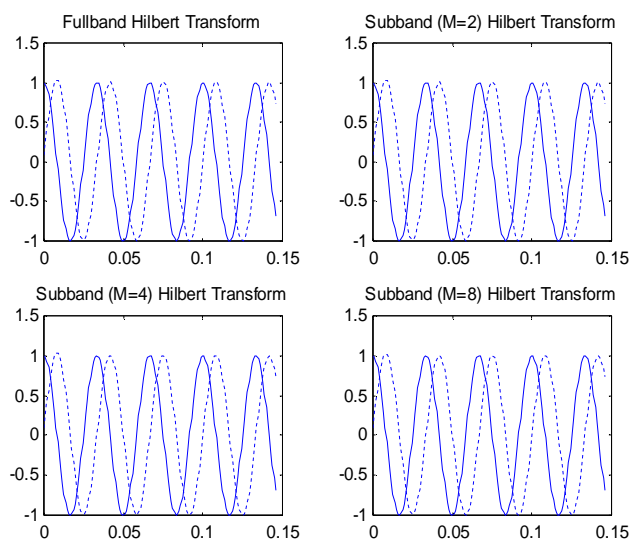

Fig. 6: Analytic signal generation.

\subsection{Single Sideband Modulation}

A SSB-modulated signal is obtained by multiplying the Hilbert transform of the information signal $x(n)$ by a sinusoid of frequency $f_{c}$ and adding (or subtracting) the result to (or from) the product of the signal $\mathrm{x}(\mathrm{n})$ and a $90^{\circ}$ phase shifted sinusoid of frequency $f_{\mathrm{c}}^{[11]}$ for upper and lower SSB signals generation, respectively. Fig. 7 shows the generation of a SSB-signal with fundamental frequency of $15 \mathrm{~Hz}$ and with transmitted carrier frequency $250 \mathrm{~Hz}$ with a sampling frequency of 1024 Hz. Both full-band and SB-Hilbert transforms with different numbers of subbands are used. The frequency spectrum of the four generated SSB-signals of Fig. 7 are shown in Fig. 8. 

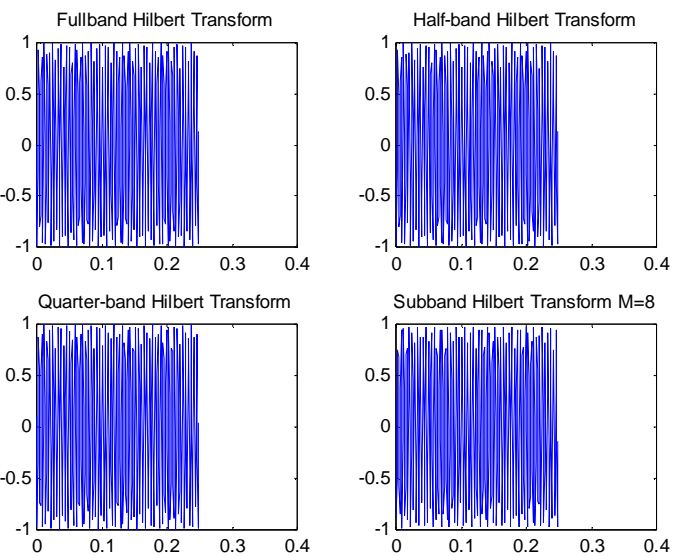

Fig. 7: Single-sideband modulation examples.
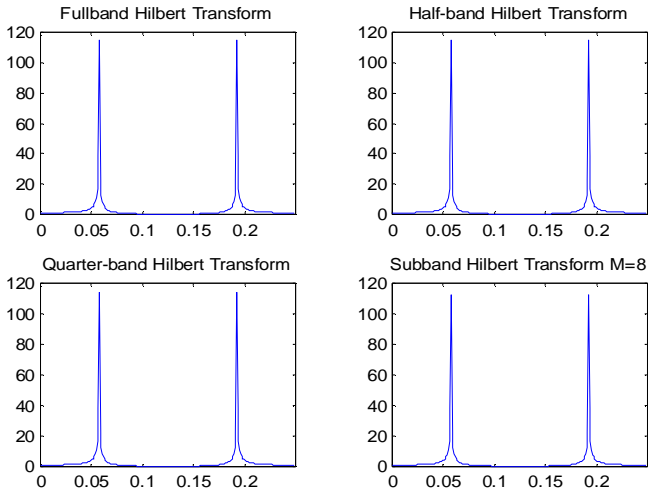

Fig. 8: Frequency spectrum of SSB examples.
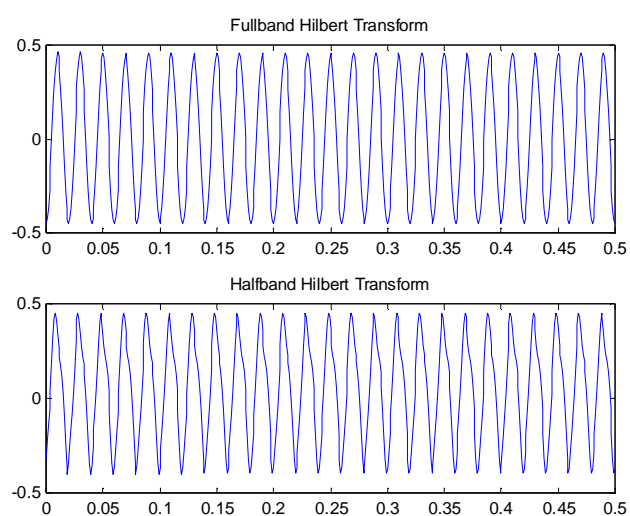

Fig. 9: Demodulated FM signal examples.

\subsection{FM Demodulation}

An FM signal may be demodulated by modulating the Hilbert transform of the waveform by a complex exponential of frequency $-f_{c}$, and obtaining the instantaneous frequency of the result ${ }^{[8]}$. In Fig. 9 a demodulated sinusoidal signal with a frequency $50 \mathrm{~Hz}$ is shown, the carrier frequency being $200 \mathrm{~Hz}$, while the sampling frequency is $1000 \mathrm{~Hz}$. Both full-band Hilbert transform and half-band Hilbert transform are used.

\section{CONCLUSION}

A new application to the subband-decomposition idea in computing the Hilbert transformation is presented. The result is a fast and approximate Hilbert transform. A reduction in complexity of about $20 \%$ to $30 \%$ in half-band and about $45 \%$ to $55 \%$ in quarter--band Hilbert transforms is obtained with respect to the fullband Hilbert transform of a discrete sequence of length $N=16$ to $N=1024$. Properties of the approximate Hilbert transform are examined with different examples. A very interesting relation between the aliasing errors of the SB-FFT used for computing the approximate Hilbert transform and the aliasing errors of the computed Hilbert transform is found. Different simulation examples of approximate Hilbert transforms with different numbers of subbands are given to describe this relation. Application examples in communication systems are also included.

\section{ACKNOWLEDGEMENT}

The author would like to thank Prof. Ulrich Heute, the head of the department of network and system theory at the university of Kiel-Germany, for his valuable comments.

\section{REFERENCES}

[1] O. Shentov; S. Mitra; U. Heute,; and A. Hossen. "Subbnad DFT-Part I: Definition, Interpretation and Extensions", Signal Processing, Vol. 41, no. 3, pp. 261-277, Feb. 1995.

[2] A. Hossen, U. Heute, O. Shentov, and S. Mitra, "Subband DFT-Part II: Accuracy, Complexity, and Applications", Signal Processing, Vol. 41, no.3, pp.279--294. Feb. 1995.

[3] S. Jung, S. Mitra, and D. Mukherjee, "Subband DCT: Definition, Analysis, and Applications", IEEE Trans. on Circuits and Systems for Video Technology, Vol.6, no.3, June 1996.

[4] A. Hossen and U Heute, "General Adaptive Sub-Band DCT", Proceedings of ECSAP-97, Prague, Czech Republic, June 1997.

[5] S. Hann," Hilbert Transforms in Signal Processing", Artech House, 1996.

[6] S. Haykin, "Communication Systems", John Wiley and Sons, 1994.

[7] A. V. Oppenheim and R. W. Schafer, "Discrete-Time Signal Processing", Prentice Hall, 1989.

[8] "Matlab Signal Processing Toolbox", The MathWorks, 1994. 
[9] A. Hossen and U. Heute, "Fully Adaptive Evaluation of Subband-DFT", Proc. of ISCAS'93, Chicago, pp.655-658, May 1993.

[10]L. Jackson, "Digital Filters and Signal processing", Kluwer Academic Publishers, 1993.

[11]J. Proakis and M. Salehi, "Communication Systems Engineering", Prentice Hall, 1994.

\section{BIOGRAPHY}

Abdulnasir Hossen received his Dr.-Ing. Degree in Digital Signal Processing from the Ruhr University of Bochum, Germany in 1994. In 1997, he completed his Post Doc. Research at the Technical Faculty of KielGermany in the same field. Then he joined the Applied Science University in Jordan as an Assistant Professor for two years. In 1999, he joined the Information Engineering Department, at Sultan Qaboos University in Oman as an Assistant Professor. Dr. Hossen's primary research interests are in digital signal processing, fast algorithms, and their applications in speech, image, radar and biomedical signal processing. 
